\title{
Average Power Prediction in Microcells
}

\author{
Silvia Ruiz-Boqué, Marta Fernandez, Marc Viladers, Ramón Agustí \\ Dep.TSC, ETS Enginyeria de Telecomunicació UPC \\ Barcelona, Spain
}

\begin{abstract}
A propagation model to estimate the average received power in an urban microcell environment has been developed. This model can be used both in Line of Sight (LOS) and Non Line of Sight (NLOS) conditions in any regular or irregular microcell structure.
\end{abstract}

\section{INTRODUCTION}

The comprehension of the propation mechanisms in different scenarios like urban, metropolitan, rural, indoor, etc. is a key factor for developing cell planning methods. They allow the the modelling and the establishment of prediction methods for attenuation (shadowing, fading statistics, penetration losses, etc.) and multipath effects (power delay profiles and delay spread) in large (macro), small (micro) and indoor (pico) cells taking into account the effects of system parameters, such as base antenna height, frequency, site location and environment.

The aim of this paper is to present some results of a path loss deterministic simulation model designed for microcells. It is based in ray tracing in three dimensional structures including building and ground reflections and diffraction and combination of reflected-diffracted rays.

The model depends on working frequency and signal polarization, on streets geometry, and on the electrical characteristics of walls and ground (dielectric properties and roughness). Once the structure is defined and transmitter position selected, the software gives the attenuation for a given position of mobile, or for a given run along streets or for the overall defined structure. The results are presented in figures (distance versus attenuation) and, if wished, streets structure is shown on computer screen and the trajectory appears in different colours corresponding to different attenuation levels.

To test the validity of the simulation several measurements in different environments in Barcelona city have been performed showing an acceptable degree of agreement [1]. Section 2 describes the theoretical expressions used for computing the path loss in regular/irregular environments. Section 3 explains the program through a description of its inputs/outputs, screen format and different options. Section 4 presents some results for different geometries. Section 5 compares some simulations with measurementes made in Barcelona.

\section{THEORY DESCRIPTION}

* LOS environment: propagation loss is dominated by interference between the direct ray $\left(\mathrm{E}_{0}\right)$, ground-reflected ray $\left(E_{g}\right)$ and building-reflected rays $\left(E_{r n}\right)$ [2]. Under this assumption the electric field can be computed by:

$$
\begin{gathered}
E=E_{o}\left[1+\frac{r_{o}}{r_{g}} R_{g} \cdot e^{-j k\left(r_{g}-r_{o}\right)}+\right] \\
{\left[+\sum_{n} \frac{r_{o}}{r_{n}} R_{n} \cdot e^{-j k\left(r_{n}-r_{o}\right)}\right]}
\end{gathered}
$$

being $r_{0}, r_{1}$ and $r_{n}$ the path lengths of direct ray, ground reflected ray and $n$-building reflected ray respectively. $R_{g}$ is the ground-reflection coefficient, $R_{n}$ is the product of $n$ reflection coefficients corresponding to $n$ wall reflections. The reflection coefficient is obtained through [3]:

$$
\begin{aligned}
& R_{H, V}=\frac{\sin \varphi-\sqrt{C_{H, V}}}{\sin \varphi+\sqrt{C_{H, V}}} \\
& C_{H}=\bar{\varepsilon}-\cos ^{2} \varphi \quad C_{V}=\frac{\bar{\varepsilon}-\cos ^{2} \varphi}{\bar{\varepsilon}^{2}} \\
& \bar{\varepsilon}=\varepsilon-j 60 \sigma \lambda \quad \rho=\exp \left[-8 \pi^{2}\left(\frac{\Delta h \cdot \sin \varphi}{\lambda}\right)^{2}\right]
\end{aligned}
$$

We assume that vertical polarization is transmitted so parallel incidence is considered for building reflections and ortogonal incidence for ground reflection. $\varepsilon$ and $\sigma$ are the permitivity and conductivity of the material and $\rho$ (rugosity) accounts for the lack of uniformity of the walls.

* NLOS environment: propagation loss is dominated by interference between building-diffracted rays and multiple combinations of reflexion and diffraction [2]. To account for diffraction phenomena for three dimensional structures the Uniform Theory of Diffraction is used (UTD) with the following assumptions: vertical polarization for trasmitted field and perfectly isotropic and conducting wedges. The diffracted fiel is then expressed as follows [4]:

$$
E_{d}(s)=E_{i}\left(Q_{d}\right) \cdot D \cdot A(s) \cdot e^{-j k s}
$$

being $\mathrm{Ei}$ the incident field on the difraction point $\mathrm{Qd}, \mathrm{s}$ the 
distance between $Q d$ and the observation point $P, A(s)$ the propagation factor describing field amplitude variations along the diffracted ray $(A(s)=1 / \sqrt{s}$ for plane wave), and $D$ the diffraction coefficient given by UTD theory as:

$$
\begin{aligned}
& D=D_{1}+D_{2}-D_{3}-D_{4} \\
& D_{1}=-\frac{\exp (-j \pi / 4)}{2 n \sqrt{2 \pi k} \cdot \sin \beta_{o}} \cdot \cot \left[\frac{\pi+\left(\phi-\phi^{\prime}\right)}{2 n}\right] \cdot F\left[k L a^{+}\left(\phi-\phi^{\prime}\right)\right] \\
& D_{2}=-\frac{\exp (-j \pi / 4)}{2 n \sqrt{2 \pi k} \cdot \sin \beta_{o}} \cdot \cot \left[\frac{\pi-\left(\phi-\phi^{\prime}\right)}{2 n}\right] \cdot F\left[k L a^{-}\left(\phi-\phi^{\prime}\right)\right] \\
& D_{3}=-\frac{\exp (-j \pi / 4)}{2 n \sqrt{2 \pi k} \cdot \sin \beta_{o}} \cdot \cot \left[\frac{\pi+\left(\phi+\phi^{\prime}\right)}{2 n}\right] \cdot F\left[k L a^{+}\left(\phi+\phi^{\prime}\right)\right] \\
& D_{4}=-\frac{\exp (-j \pi / 4)}{2 n \sqrt{2 \pi k} \cdot \sin \beta_{0}} \cdot \cot \left[\frac{\pi-\left(\phi+\phi^{\prime}\right)}{2 n}\right] \cdot F\left[k L a^{-}\left(\phi+\phi^{\prime}\right)\right]
\end{aligned}
$$

being $\mathbf{k}$ the wave propagation factor, $\beta_{0}$ the incident angle over the wedge, and $\phi$ and $\phi^{\prime}$ are the angles of the incident and reflected rays measured in a plane ortogonal to the wedge and respect the same side of the wedge. The internal angle of the wedge is $\alpha=(2-n) \pi$. F[kLa] is the Fresnel transition function for solving indetermination problems in transition regions. The symbols + and - stand for side " 0 " or side " $n$ " of the wedge iluminated respectively. $L=s \cdot \sin ^{2} \beta_{o}$ for incident plane waves.

$$
\begin{aligned}
& F(x)=2 j \sqrt{x} \cdot e^{j x} \cdot \int_{\sqrt{x}}^{\infty} \exp \left(-j t^{2}\right) d t \quad x \geq 0 \\
& F(x)=F^{*}(|x|) \quad x<0 \\
& a^{ \pm}\left(\phi \pm \phi^{\prime}\right)=2 \cos ^{2}\left[\frac{2 n \pi \cdot N^{ \pm}-\left(\phi \pm \phi^{\prime}\right)}{2}\right]
\end{aligned}
$$

being $\mathrm{N}$ the integer which better follows:

$$
2 \pi n \cdot N^{+}-\left(\phi \pm \phi^{\prime}\right)=\pi \quad 2 m \cdot N^{-}-\left(\phi \pm \phi^{\prime}\right)=-\pi
$$

The total propagation loss is obtained by adding the contributions of each ray taking into account that they are complex parameters. The simulation program offers also the possibility of adding directly the power contribution of each ray in order to smooth the curves [5].

\section{SIMULATION PROGRAM}

* Building and streets layout: the geometry is introduced into the simulation program as follows: the map of the microcellular environment is digitalized through the use of AutoCad program and a digitalizer tablet. While so doing we obtain a file in AutoCad format containing vectors with two dimensional coordinates for each building or set of buildings. This file is read by a $\mathrm{C}$ program which allows for each vector to introduce building characteristics (heigh, permitivity, conductivity and rugosity). This structure defines completely the environment where propagation losses will be calculated.

* Obtaining the propagation loss: The program for obtaining the propagation loss is also written in $\mathrm{C}$ lenguage and structured in menus. First of all the user sees in the computer screen the digitalized map with the different buildings represented in different colours according to their category (concrete wall with few windows, concrete wall with windows, glass wall, and irregular building wall this is with several balconies and sections of the wall inclinated, etc.). Then the frequency (usually 900 or $1800 \mathrm{MHz}$ ) and the ubication of base antena are selected (including heigh).

The user has then the possibility to choose between two options: obtaining the propagation loss for a fixed position of the mobile or for a nun. Whatever the option the coordinates are always introduced graphically by using the mouse and the program translates to $x, y, z$ values.

Then the user selects the limits for the rays contributing to the received power. The program allows to choose the number of reflections and diffractions considered and also different combinations of diffraction and reflection. It allows also to check the contribution of propagation ways different from the principal (through double or triple diffraction and reflection-reflection-reflection). This "propagation characteristics" can be saved in a file for being used again. The possibility of activate/desactivate the contributions allows a separate study of each one and to state its influence over the received power.

- number of reflections along the same street (up to 10)

- number of diffractions (up to 3 )

- reflection-diffraction: yes (includ.) or no (not incl.)

- diffraction -reflection (yes or no)

- reflection-diffraction-reflection (yes or no)

- refl.-refl. in different streets (yes or no)

- refl.-refl.-refl. in different streeets (yes or no)

Contribution of ways different from the principal one and the effect of multiple diffractions have been found to be negligible. To reduce the program running time, once the trajectory of the mobile has been defined, the user "helps the program" selecting with the mouse the building vectors which can play a role in the evaluation of propagation losses. If we do not do that the program has to consider all the vectors and all the combinations between vectors meaning that we should wait for hours before obtaining the result for a single street with LOS. 


\section{SIMULATION RESULTS}

When a regular structure is considered the building wedge has an internal angle $\alpha=\pi / 2$ and then $n=1.5$. Two different runs have been studied in fig. 1 (a) is a run along an avenue ortogonal to the LOS street and allows to study what happens when the mobile turns a corner, (b) is a nun along a street paralel to the LOS street allowing to study what happens when the mobile turns two corners.

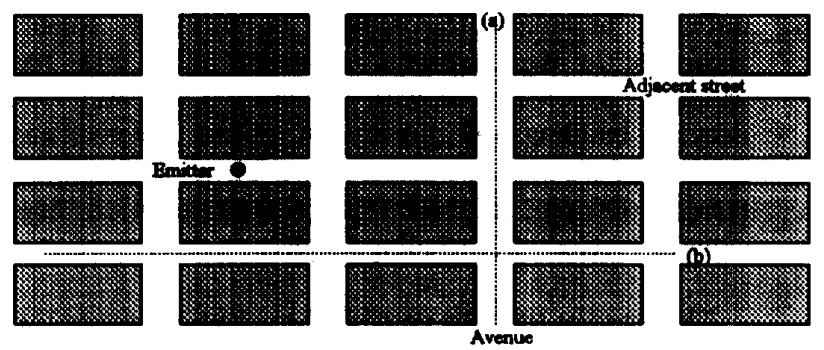

Fig.1 Regular structure and considered runs.

* Run along an avenue: The direct and ground reflected rays contribute only around the intersection with the street where base st. is ubicated (Fig.2). Its influence increases when the base st. gets closer to the avenue or widening the street containing the base station. In this two cases the transition from LOS to NLOS is smoother. Reflections on buildings also contribute to the field and extend their influence to the NLOS zone as can be shown in fig.3.
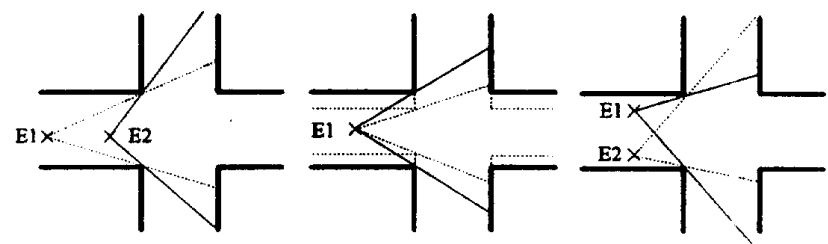

Fig. 2 LOS contribution under different conditions

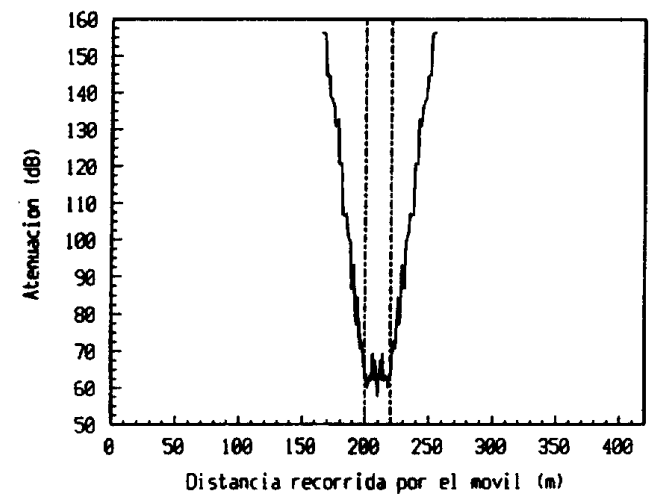

Fig.3 LOS fields contribution over the intersection
Results considering only diffr. are in fig.4. Attenuation along the avenue is almost constant. A similar curve is obtained when multiple refl. in LOS street followed by diffraction is considered.Path loss due to corner diffr. and refl. along avenue is in fig.5. Contribution to LOS zone is zero and attenuation level along the avenue is similar to previous cases but with fluctuations. refl-diffr-refl case is similar with the particularity that attenuation increases along the avenue. Attenuation level increases quickly as the mobile goes deeper in the avenue when refl.-refl. in LOS street-NLOS avenue is considered (fig.6).
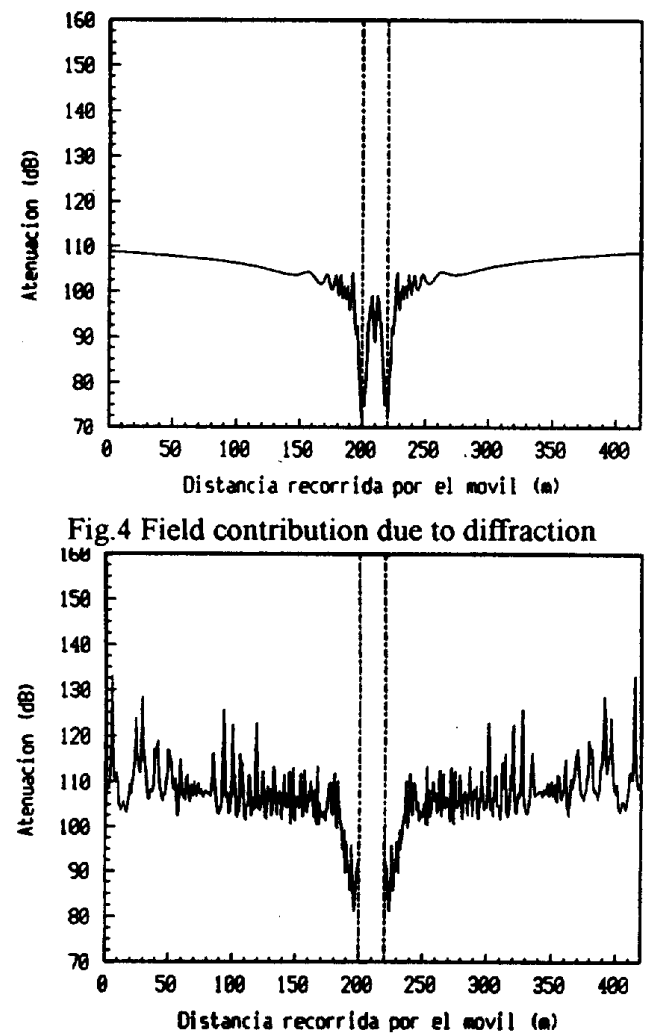

Fig. 5 Contribution of difraction-reflection phenomena

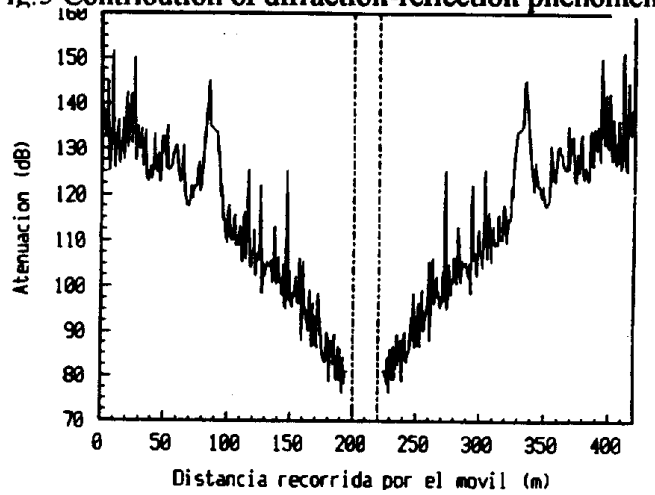

Fig. 6 Contribution of reflection-reflection fields 
The rest of contributions show no incidence to the total field, represented in fig. 7 and in fig. 8 by adding all terms.

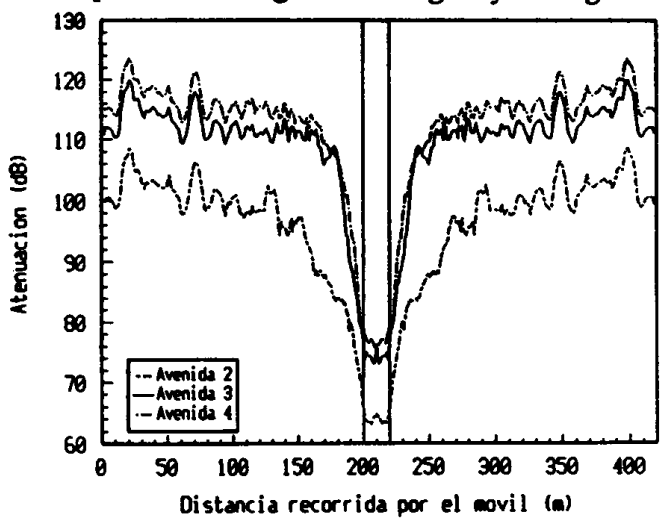

Fig. 7 Total path loss for three avenues

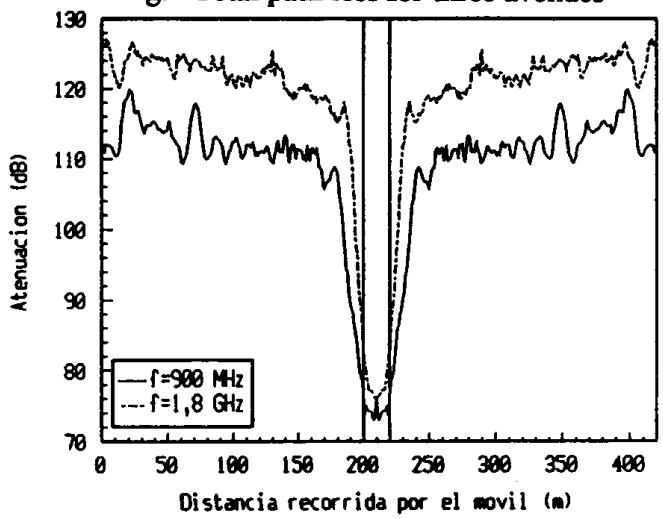

Fig. 8 Total path loss in the avenue for two frequencies

Run along a street: We have studied the contributions to the field along a street being in this case the mobile always in NLOS condition (direct ray, building reflections and ground reflected ray do not contribute). Moreover the rays due to difr., refl.-difr., difr.-refl., refl.-difr.-refl. and refl.-refl. show an important contribution but only in the intersections with the avenues. In the rest of the street path loss is given mainly by double diffraction and refl.refl.-refl. Path loss is represented in fig. 9 and in fig. 10 .

Simulations allow to state that antenna height is not relevant therefore the simulation could be simplified to a bidimensional study. A change in the width of streets or avenues implies a widening/shortening of the flat zones corresponding to intersections in the run along the avenue but the attenuation level remains similar (test with 20 and $40 \mathrm{~m}$ wide streets and avenues). In the run along a street a widening in the streets or in the avenues (20 to $40 \mathrm{~m})$ implies a decrease in attenuation level of about 8-10 dB in the intersections being the rest of the curve very similar. Results when only 3 wall refl. are considered are almost identical to the ones with 10 refl. allowing a considerable reduccion of computing time maintainig accuracy.
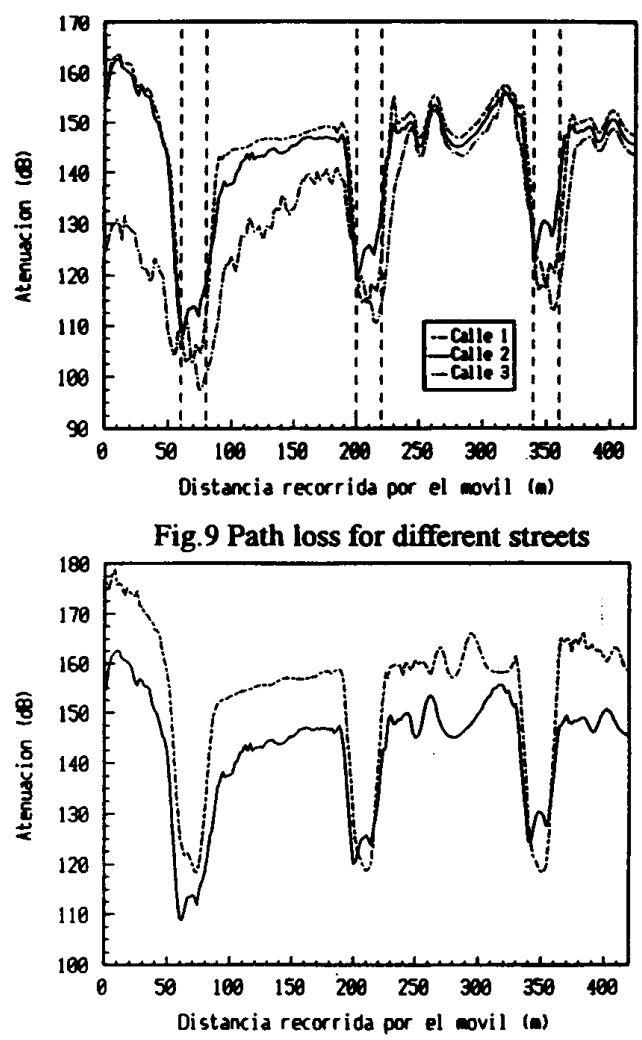

Fig.10 Path loss in a street for two frequencies

* Irregular structure: a similar study has been developed for different street orientation to validate the results. Obtained results are easily predictable being the main difference from regular structure the fact that LOS contribution is strongly dependent of the angle between the street containing the transmitter and the NLOS street. From the point fo view of the program no simplification over the general expression for the diffraction coefficient can be considered and then, it is necessary to known (introduced as a parameter) the different angles between the two vectors which shape the corner.

\section{COMPARISON WITH MEASUREMENTS}

Measures were made at $1800 \mathrm{MHz}$ in Barcelona metropolitan area in LOS and NLOS conditions. Next figures show the map corresponding to a nu, and measured and simulated attenuation. Differences can be due to several reasons: building materials different from simulated ones, distance for averaging the attenuation are different (about $8 \mathrm{~m}$ in the measures and $3 \mathrm{~m}$ in simulation), being the structure nearly regular we have 
considered corners of $90^{\circ}$ being then the attenuation valleys sharper in the simulation. Unfortunately we have had no time to test our simulation program for irregular structures with real environment and with measurements, but this will be presented at the conference meeting.
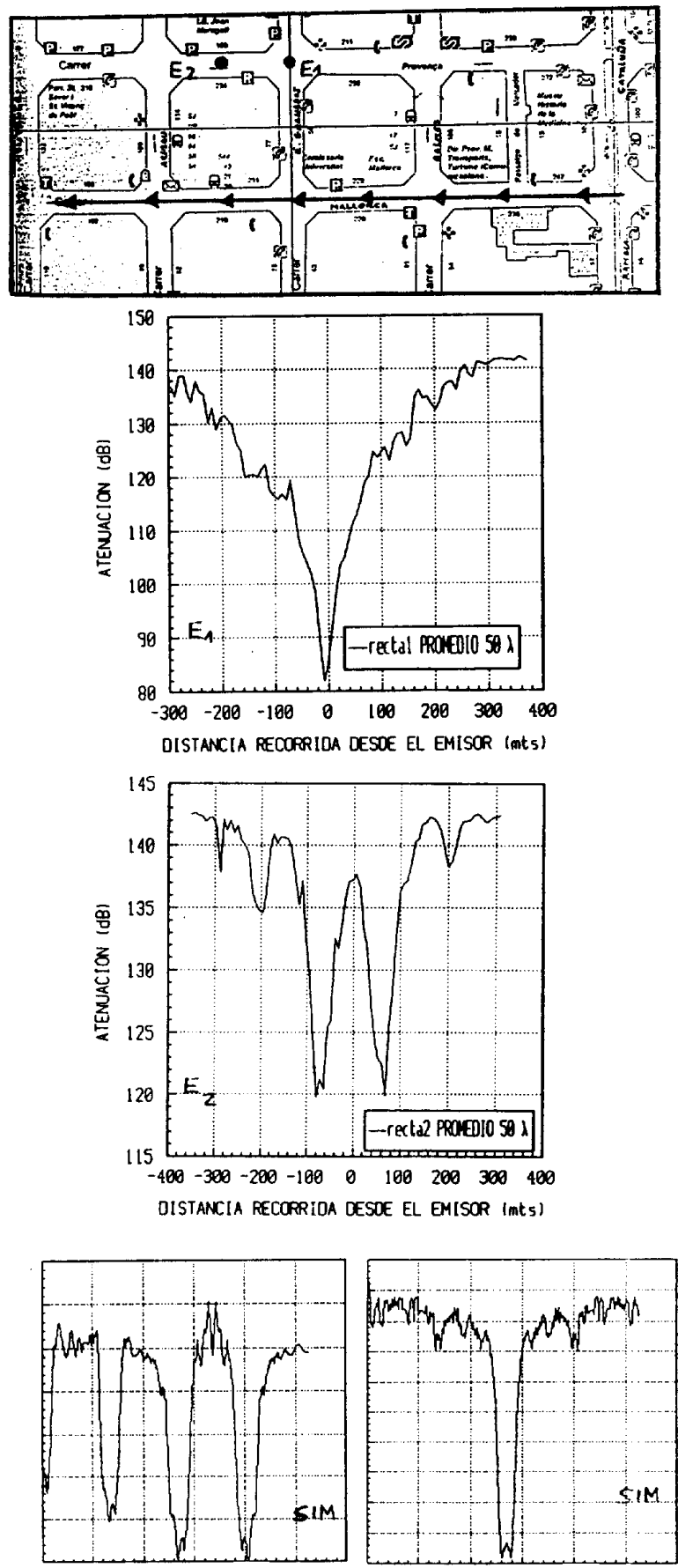
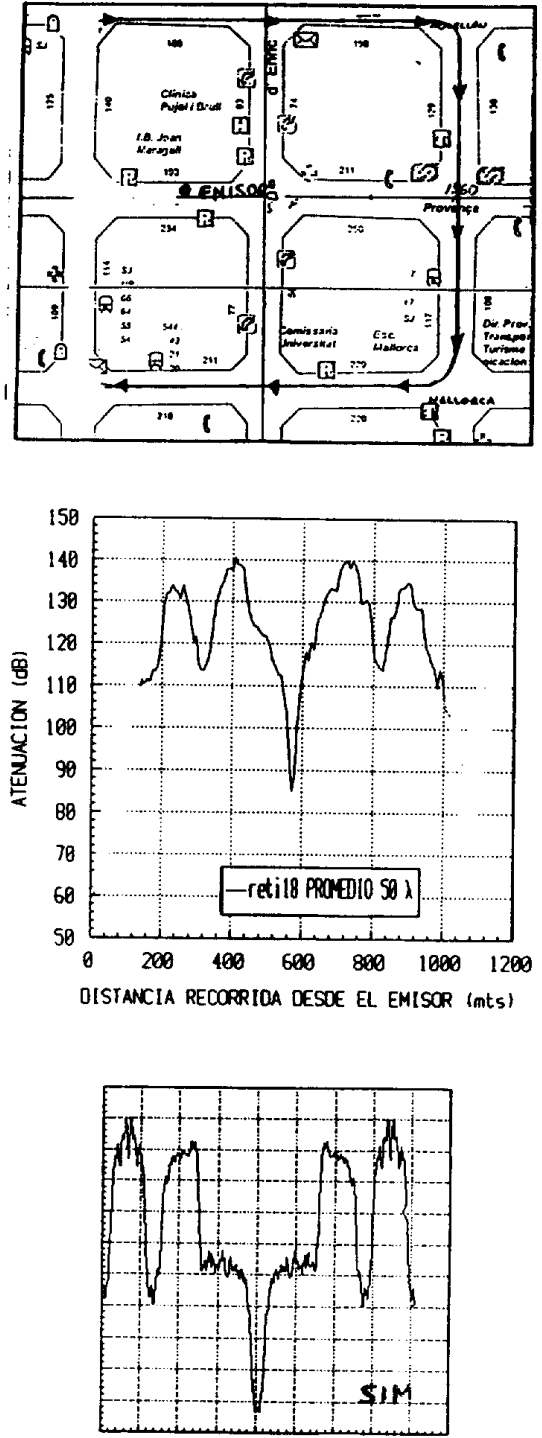

\section{REFERENCES}

[1] I.Espinel, J.Lozano,S.Ruiz,_Boqué "Propagation measures and models for microcells at $1900 \mathrm{MHz}^{\prime \prime}$, Docs. COST231 TD(93), no.17, Barcelona , january 1993.

[2] Chia, R.Steele, E.Green and A.Baran "Propagation and bit error ratio measurements for a microcellular system", IERE, Vol.57, No.57, No.6, pp.s255-s266, Nov. 1987

[3] L.Bohitias, "Propagation des ondes radioelectriques dans l'environment terrestre, Bordas et CNET, Paris 1983.

[4] R.Kouyoumjian and P.Pathak "A Uniform Geometrical heroy of Diffraction for and Edge in a Perfectly Conductiong Surface", Proc.IEEE, Vol.62, no.11, pp.1448-1461, Nov. 1974.

[5] J. Wagen adn K.Rizk "Simulation of radio wave propagation in urban microcellular environments" Doc.COST 231 TD(93), no. 